Note: This paper is a pre-print version of an article published in Addictive Behaviors, and is not the final copy-edited version.

Please cite this paper as:

Qureshi, A., Monk, R. L., Pennington, C. R., Wilcockson, T. D., \& Heim, D. (2019).

Alcohol-related attentional bias in a gaze contingency task: Comparing appetitive and nonappetitive cues. Addictive Behaviors, 90, 312-317. doi: 10.1016/j.addbeh.2018.11.034

\title{
Alcohol-related attentional bias in a gaze contingency task: Comparing appetitive and non-appetitive cues
}

Adam Qureshi 1*, Rebecca L. Monk ${ }^{1}$, Charlotte R. Pennington ${ }^{2}$, Thomas D.W.

Wilcockson $^{3}$, Derek Heim ${ }^{1}$

${ }^{1}$ Edge Hill University, St Helens Rd, Ormskirk, UK, L39 4QP

${ }^{2}$ University of the West of England

${ }^{3}$ Lancaster University

*Corresponding author: Email qureshia@edgehill.ac.uk

Declarations of interest: none

The authors declare no conflicts of interest and declare that this paper is not under review or in press at any other journal, nor will it be submitted elsewhere until the completion of the decision making process. 


\begin{abstract}
Background: Social drinkers attend automatically to alcohol-related cues compared to nonalcohol related cues on tests of inhibitory control. Moreover, attentional bias for alcoholrelated cues varies between problem and non-problem drinkers. Aim: To examine attentional bias towards alcoholic and non-alcoholic appetitive cues between problem and non-problem drinkers. Method: Forty-one university students ( 9 male, 32 female; $M$ age $=21.50$ ) completed an eye-tracking gaze contingency paradigm, measuring the number of times participants looked at peripherally and centrally located stimuli (break frequency), when instructed to maintain focus on a target object. Stimuli consisted of appetitive alcohol-related (e.g., beer), appetitive non-alcohol-related (e.g., orange juice) and non-appetitive (e.g., washing liquid) stimuli. Participants were split into non-problem $(M$ AUDIT $=3.86)$ and problematic drinkers $(M$ AUDIT $=11.59)$. Results: Problematic drinkers had higher break frequencies towards peripheral appetitive stimuli than towards non-appetitive stimuli, while break frequency was equivalent between appetitive cues presented centrally (alcohol and nonalcohol-related). In contrast, there were no differences in break frequency across stimuli type or cue presentation location (central or peripheral) for non-problem drinkers. Conclusion: In contrast to non-problem drinkers, respondents displaying more problematic consumption practices may find it more difficult to inhibit eye movements toward appetitive stimuli, particularly when in peripheral vision. This may suggest that attentional biases (as measured in terms of overt eye movements) in problem drinkers may be most powerful when the alcoholic and appetitive stimuli are not directly in field of view. An uncertainty reduction process in the allocation of attention to appetitive cues may help explain the patterns of results observed.
\end{abstract}

Key words: Inhibitory Control; Attentional Bias; Alcohol; Appetitive; Gaze Contingency 


\section{Introduction}

Inhibitory control can be defined as people's ability, or capacity, to control their actions, attention or responses, and is believed to be important during attempts to regulate behaviour (Simpson \& Riggs, 2006). Accordingly, there are well established links between inhibitory control, attentional bias, and substance use and these have been demonstrated via the use of a whole host of tasks including the Emotional Stroop task (e.g., Cox, Yeates, \& Regan, 1999), the Go No Go Association Test (e.g., Fleming \& Bartholow, 2014), the Stop Signal Reaction Test (e.g., Li, Luo, Yan, Bergquist \& Sinha, 2009) and the anti-saccade paradigm (e.g., Jones $\&$ Field, 2015). The current research aims to examine failures in inhibitory control owing to attentional bias and utilising both appetitive alcohol-related, non-appetitive alcohol-related and non-appetitive (neutral) cues.

Whilst inhibitory control is often viewed as a trait ability that is independent of contextual influences (Leotti \& Wager, 2010), an alternative view suggests that this form of executive control is a limited, consumable resource which can be depleted in situations that involve resisting temptation (Muraven \& Baumeister, 2000). Inhibitory control fluctuations may therefore be similar to mental fatigue and be associated with contextually induced changes to people's motivations and priorities (Inzlicht \& Berkman, 2015). Understanding this process may be crucial to improving intervention approaches which seek to improve such inhibitory control levels (e.g., Jones et al., 2016). As such, overall impairments in inhibitory control may be associated with problem consumption while contextual fluctuations in one's inhibitory capacity may be associated with elevated but non-problematic substance use. Specifically, alcohol-related visual cues (e.g., Christiansen, Cole, \& Field, 2012; Kreusch et al., 2013; 2017; Weafer \& Fillmore, 2015; 2012 - c.f., Jones, Rose, Cole \& Field, 2013; Nederkoorn et al., 2009; Rose \& Duka, 2008), sounds (e.g., Qureshi et al., 2017) and olfactory cues (Monk et al., 2016) have been shown to impair inhibitory control which may, in turn, impact consumption.

Recently, a gaze contingency task has been developed as a novel method for measuring inhibitory control failure as a direct consequence of attentional bias. This paradigm assesses the compulsive nature of attention towards certain stimuli and allows researchers to quantify how distracting alcohol-related stimuli are (Wilcockson \& Pothos, 2015). Specifically, it measures ability to inhibit orientation towards peripheral alcohol-related stimuli when focusing on a specified target. In accordance with the predominant literature in this area, increased alcohol use was found to be associated with the number of 'attentional breaks' recorded in 
response to alcohol-related stimuli (break frequency). Put simply, people who consume more alcohol seem to find alcohol-related stimuli more distracting or feel more compelled to move look towards it (Wilcockson \& Pothos, 2015). Within the original study, the authors did not conduct their analyses on peripheral and central stimuli separately. They instead looked at overt attentional shifts overall for all stimulus locations. However, it is possible that inhibitory control may be increased for centrally located stimuli because they can be processed much more easily with covert attention (e.g., Hogarth et al., 2008). The current study therefore separates peripheral from central stimulus locations in order to measure whether inhibitory control is affected by the ability to covertly attend to stimuli.

The current research also builds on the existing literature by examining more closely the appetitive nature of the stimuli that are used within many studies of inhibitory control (see Monk, Qureshi, Pennington, \& Hamlin, 2017 for more description of the nature and use of appetitive vs non appetitive cues). For example, Wilcockson and Pothos (2015) assessed attentional bias by examining the difference score between mean break frequency ${ }^{1}$ to alcoholrelated (e.g., a pint of beer) and non-alcohol-related neutral cues (e.g., office equipment). Indeed, there has been extensive research which does so and suggests an increase in disinhibition in response to alcohol-related cues (e.g., pictures of bottles of beer) in contrast to neutral stimuli (e.g., a stapler - Kreusch et al., 2013, or a stool, bus or umbrella - Jones \& Field, 2015). However, by contrasting appetitive and non-appetitive cues (non-palatable/ingestible), it is unclear from the existing literature whether utilising other consumable products would elicit the same findings. To wit, more recent research has demonstrated the importance of using adequate control stimuli (see Adams, Ataya, Attwood, \& Munafò, 2014). A recent empirical study demonstrates further that decreases in inhibitory control in response to alcohol-related cues on a Stop-Signal Task might generalise to other appetitive liquids but not to non-appetitive cues (Monk, Qureshi, Pennington, \& Hamlin, 2017). As such, it remains to be seen whether observed findings of bias to alcohol-related stimuli may be attributable, at least in part, to the appetitive content of the stimuli used, as opposed to comparing alcohol versus non-alcohol related appetitive cues.

\footnotetext{
${ }^{1}$ i.e., the average number of times participants looked away from the fixation target and towards the distractor cues
} 
The current research therefore aims to examine failures in inhibitory control owing to attentional bias and utilising both appetitive alcohol-related, non-appetitive alcohol-related and non-appetitive (neutral) cues. Using the gaze contingency paradigm of Wilcockson and Pothos (2015), this research will also consider both overt and covert forms of attention by assessing attention towards both central and peripherally occurring stimuli. In so doing, we seek to further our understanding of alcohol-related disinhibition.

\section{Method}

\section{Participants}

Forty-one participants were recruited from a UK university ${ }^{1}$ ( 9 male; Mage $=21.50, S D=6.61$, overall $\mathrm{MAUDIT}=7.32, \mathrm{SD}=4.97)$ and were reimbursed with course credits. Post-hoc power analyses suggested that with the power to detect the observed effect size of $\eta_{p^{2}}^{2}=.07$ for the three-way interaction, with alpha set at 0.05, was .97.

Participants were divided post hoc using a median split (median AUDIT score $=7.5$ ) of their scores on Alcohol Use Disorders Identification Test (AUDIT; Saunders et al., 1993. Those with scores above 8 on the AUDIT are classified as harmful drinking patterns (Saunders et al., 1993) and as such, we classified participants as non-problem drinkers ( $n=23$, Maudit $=3.86)$ or problem drinkers $(n=18$, Maudit $=11.59)$. There was a significant difference in AUDIT scores between the groups $\left(\mathrm{F}(1,36)=57.64, p<.01, \eta_{p}^{2}=.62\right.$; see Table 1). There was no difference in the gender composition of the groups $\left(X^{2}(1, N=41)<.01, p=.97\right)$.

\footnotetext{
${ }^{1}$ Two participants were excluded from the final analysis due to their AUDIT scores being 0 .
} 
Table 1. AUDIT, age and gender by consumption type and overall

\begin{tabular}{cccccc}
\hline \multirow{2}{*}{ Group } & Age $(M(S D))$ & AUDIT $(M(S D))$ & Male & Female & $\mathrm{N}$ \\
\hline $\begin{array}{c}\text { Non-problem } \\
\text { drinkers }\end{array}$ & $22.86(8.49)$ & $3.86(1.96)$ & 5 & 18 & 23 \\
$\begin{array}{c}\text { Problem drinkers } \\
\text { Overall }\end{array}$ & $19.82(2.33)$ & $11.59(4.14)$ & 4 & 14 & 18 \\
& $21.50(6.61)$ & $7.32(4.97)$ & 9 & 32 & 41 \\
\hline
\end{tabular}

\section{Design}

The main task used a mixed factorial ANOVA, with within-participants factors of stimuli type (alcoholic $\mathrm{x}$ non-appetitive $\mathrm{x}$ non-alcoholic) and stimuli location (central $\mathrm{x}$ peripheral), and a between-subjects factor of alcohol consumption group (non-problem $\mathrm{x}$ problem drinkers), resulting in a $3 \times 3 \times 2$ ANOVA.

Stimuli and Materials

The main task was a gaze contingency task which was run on an EyeLink Desktop 1000 eye-tracker, and designed using Experiment Builder (SR Research). This built on the study designed by Wilcockson and Pothos (2015) ${ }^{1}$ and introduced participants to three categories of visual stimuli; alcoholic appetitive, non-alcoholic appetitive, and non-appetitive cues (see Figure 1 for examples). In this task, a fixation target was presented on-screen and participants were required to always look this and not at distractor stimuli. Distractor stimuli were presented within any one of eight regions presented on the computer screen (see Figure 2). These were classified as either 'central' distractors positioned close to the fixation target or 'peripheral' distractors positioned further away (see Figure 3). The fixation target and distractor stimuli were designed to be equally visually salient.

\footnotetext{
${ }^{1}$ Which originally combined alcoholic stimuli and neutral stimuli consisting of stationary items
} 

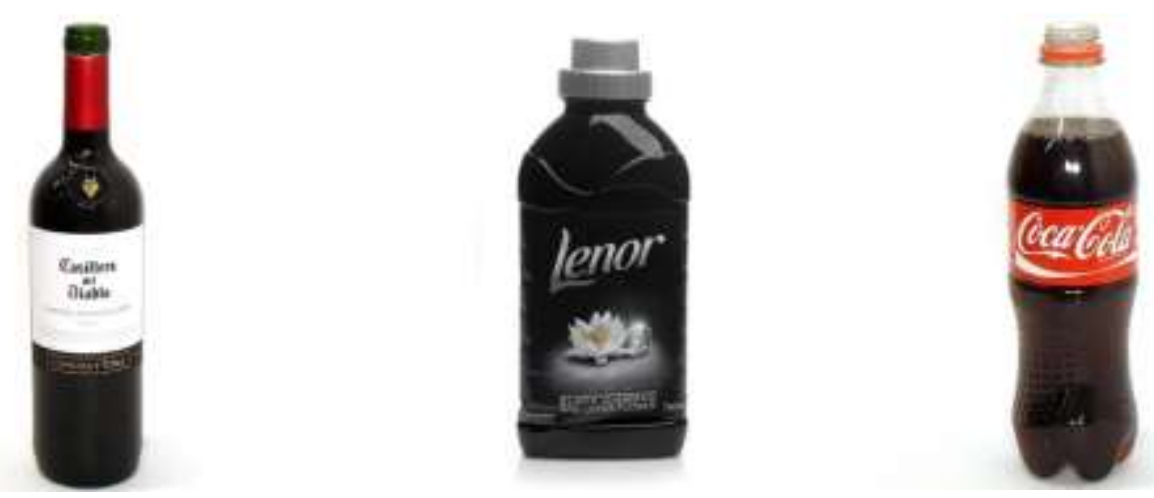

Figure 1. Examples of (L-R) appetitive alcoholic, non-appetitive and appetitive non-alcoholic stimuli

All stimuli were equivalent in size (set to 500 x 500 pixels), though when presented on the screen the visual angle was .08 radians. 90 images in total were included in this experiment, 30 each of non-alcoholic appetitive stimuli, alcoholic appetitive stimuli and non-appetitive stimuli. These were matched on valence, arousal, angles of objects, luminance and colour using pilot testing. Specifically, branded images of alcoholic (beer, wine, and spirits) and non-alcoholic drinks (water, soft drinks) were taken from the Alcohol Beverage Picture Set (ABPS); Pronk et al., 2015) and these were matched against non-appetitive branded products, including washing up liquid and cleaning items, which were assessed during pilot testing ${ }^{1}$. Pilot results are shown in Appendix A.

\footnotetext{
${ }^{1}$ A total of 78 participants ( 31 male; $M$ age $=19.41, S D$ age $=5.32$ ) were tested using a similar design to that of Pronk et al. (2015). Participants were asked to rate 72 images (24 images each of washing-up liquid, fabric softener and disinfectant) on parameters to match stimuli across categories (recognition, valence, arousal, use/consumption, knowledge and control). Valence, arousal and control were included as per the International Affective Pictures Image System (Lang et al., 2005). Images were presented using Bristol Online Surveys (BOS) and remained on-screen until the participant responded. Nine answer options were provided for each image, with Self-assessment Manikins above every other answer option (see Suk, 2006, p. 206; arousal and control based on Lang, 1980). Answers were scored from -4 (left-hand side) through to +4 (right-hand side), with negative values representing more negative affect, relaxation and being controlled, and positive values representing more positive affect, excitement and having control. The urge to use or consume the liquid was also assessed by a 9-point bipolar scale (from $-4=$ "absolutely not" to $+4=$ "Very much"). The final two items determined whether the participant knew the product and if they had ever used it, both using dichotomous yes/no responses.
} 


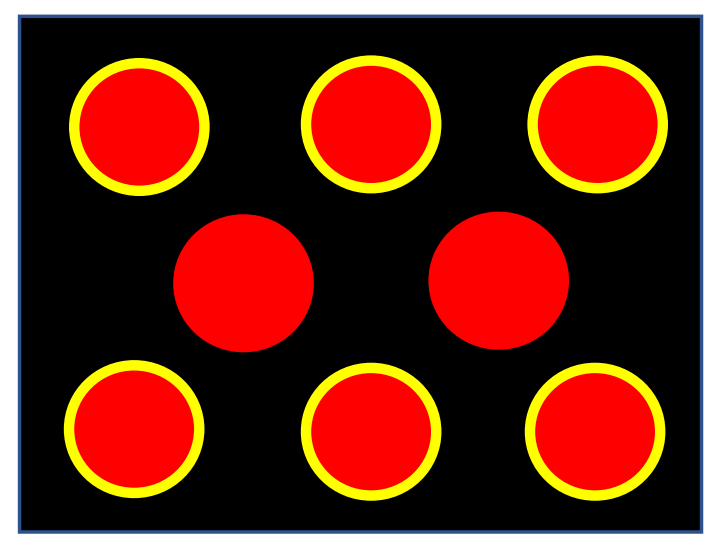

Figure 2. Fixation target and distractor positions. Red circles indicate possible distractor locations, yellow borders show positions the fixation target could also appear in.
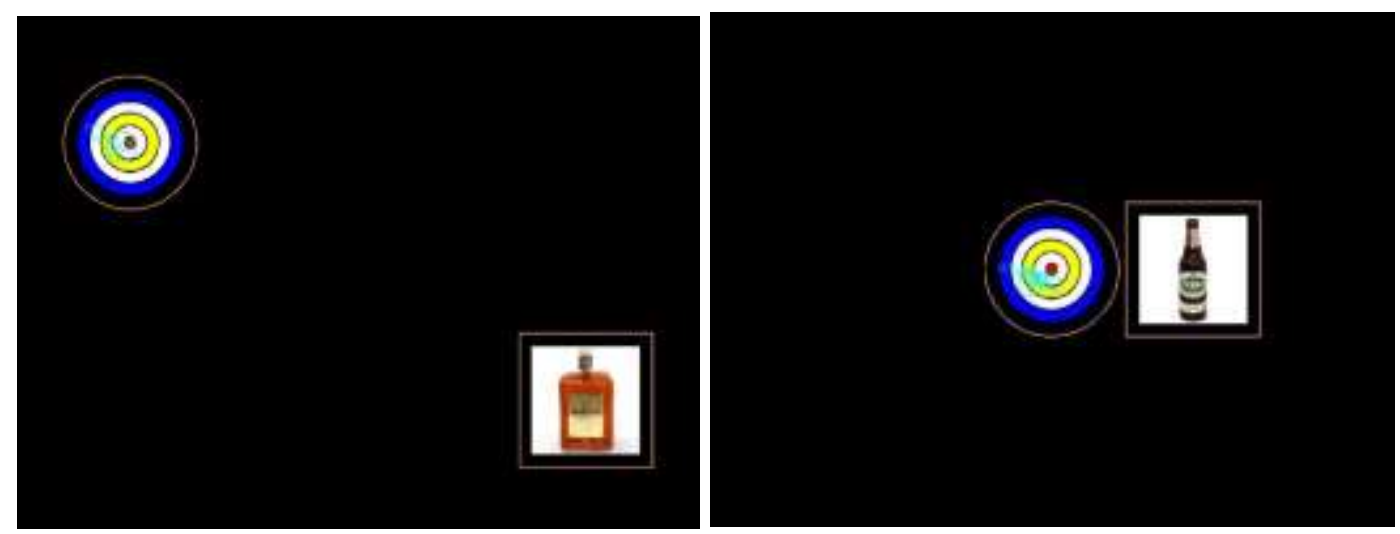

Figure 3. Examples of peripheral (L) and central (R) distractor stimuli (borders = areas of interest).

Procedure

Ethical approval was granted by the governing institution's ethics committee. Participants were recruited via online advertisements asking for interested volunteers. Those who were interested contacted the researcher, provided informed consent and completed an online AUDIT questionnaire prior to attending the experimental session, to avoid alcohol-related priming of the questionnaire content. When participants arrived at the eye tracking laboratory, following briefing and the provision of informed consent, participants were seated $57 \mathrm{~cm}$ away from the computer using a head mount. Their eye-movements were calibrated on the eye-tracker and 
they were instructed to look at the colour target presented on the screen and to ignore the distractors then presented.

After participants gazed at the fixation target for a set interval of $1000 \mathrm{~ms}$, a distractor stimulus (either alcoholic, non-alcoholic or non-appetitive) appeared (one per trial). If the participant then looked at the distractor stimulus, this would disappear. If participants returned their gaze to the fixation target for $10 \mathrm{~ms}$ (less than one frame on a $60 \mathrm{~Hz}$ ) monitor, the distractor would reappear. The fixation target was displayed for $5000 \mathrm{~ms}$ in total, so the maximum onscreen time for the distractor was $4000 \mathrm{~ms}$. A total of 91 trials were presented $(30 \mathrm{x}$ nonalcoholic appetitive distractors, $30 \mathrm{x}$ alcoholic appetitive distractors, $30 \mathrm{x}$ non-appetitive distractors, $1 \mathrm{x}$ initial blank distractor), split into blocks of 31, 30 and 30 trials. The number of times that participants looked at the distractor stimuli was measured and taken as the dependent variable. The study took approximately 15 minutes in total. After completion of the study, participants were debriefed.

\section{Results}

A 3 (Stimuli type: Alcoholic, non-alcoholic, non-appetitive) x 2 (Stimuli location: Central, Peripheral) x 2 (Alcohol consumption group: non-problem drinkers, problem drinkers) mixed-design ANOVA was conducted on the proportion of gaze fixations made to the distracter stimuli (mean break frequency). Stimuli type and location were input as withinfactors and alcohol consumption group was input as a between-factor.

A main effect of stimuli type was found $\left(\mathrm{F}(2,78)=3.59, p=.03, \eta_{p}^{2}=0.08\right)$, with higher mean break frequency to the stimuli for non-alcoholic stimuli compared to nonappetitive stimuli $(p=.04)$. As expected, there was also a main effect of stimuli location (F ( 1 , $\left.39)=15.22, p<.01, \eta_{p}^{2}=.28\right)$, with higher mean break frequencies to central stimuli $(p<.01$, $\left.\eta_{p}^{2}=.28\right)$. There was an interaction between stimuli type and stimuli location $(\mathrm{F}(2,78)=4.10$, $\left.p=.02, \eta_{p}^{2}=.10\right)$, and a 3-way interaction between stimuli type, stimuli location and alcohol 
consumption group $\left(\mathrm{F}(2,78)=3.11, p=.05, \eta_{p}^{2}=.07\right)$. The three-way interaction was explored using simple main effects with Bonferroni corrections ${ }^{1}$ (see Figure 4).

For problematic drinkers, there was a higher mean break frequency to centrallylocated non-appetitive stimuli compared to centrally-located alcoholic stimuli $(p=.03$; mean difference $=.035 ; \eta_{p}^{2}=.16$ ), with no difference between central alcoholic and non-alcoholic stimuli $\left(p>.93, \eta_{p}^{2}<.07\right)$. For peripheral stimuli, there were higher break frequencies to non-alcoholic stimuli compared to non-appetitive stimuli $(p=.04$; mean difference $=.032$; $\left.\eta_{p}^{2}=.16\right)$, and marginally higher mean break frequencies to alcoholic stimuli compared to non-appetitive stimuli $\left(p=.054\right.$; mean difference $\left.=.025 ; \eta_{p}^{2} .12\right)$. There were no differences between alcoholic and non-alcoholic stimuli $(p=1.00)$. There were no differences for nonproblematic drinkers (all $p$ 's $>.09, \eta_{p}^{2}<.12$ ).

For the low consumption group, there was a higher mean break frequency to central stimuli compared to peripheral stimuli for all stimuli types $(p$ 's $<.02$; mean differences: alcoholic $=.034, \eta_{p}^{2}=.18$; non-appetitive $=.037, \eta_{p}^{2}=.17$; non-alcoholic $\left.=.032, \eta_{p}^{2}=.14\right)$. For problematic drinkers, there were higher mean break frequencies to non-appetitive central stimuli compared to non-appetitive peripheral stimuli $\left(p<.01\right.$; mean difference $=.053 ; \eta_{p}^{2}=$ $.24)$, with no other differences $\left(p\right.$ 's $\left.>.60, \eta_{p}^{2}<.01\right)$. Whether located centrally or on the periphery, no difference for any stimuli type was found on for mean break frequencies between the consumption groups ( $p$ 's $\left.>.15, \eta_{p}^{2}<.05\right)$.

\footnotetext{
${ }^{1}$ These were implemented via SPSS syntax. As Bonferroni adjustments are generally considered to be conservative, Holm's sequential Bonferroni procedure was also carried out using the Holm-Bonferroni Sequential Correction: An EXCEL Calculator (Justin Gaetano, 2013). There were no differences in the results found.
} 


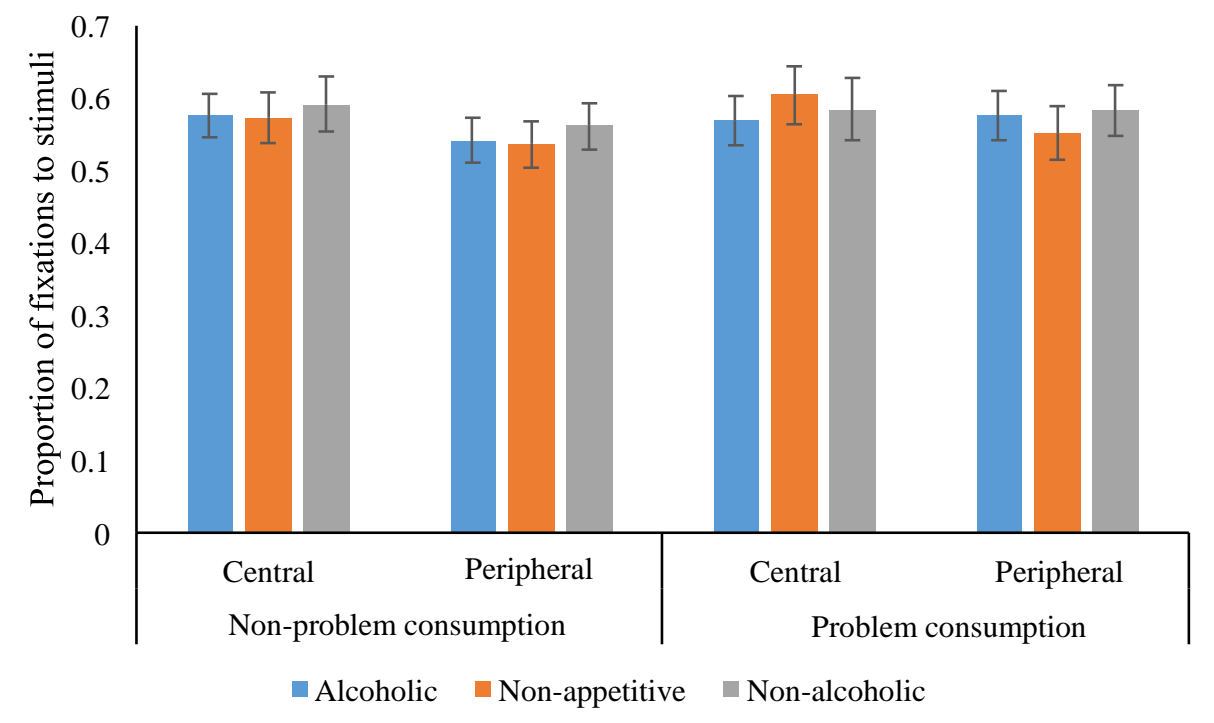

Figure 4. Proportion of fixations to stimuli by location, alcohol consumption group and type (bars $-95 \%$ confidence intervals)

Analyses including pilot measures of valence and control as covariates are shown in Appendix A, but do not significantly affect the key finding of higher mean break frequencies to appetitive (in this case alcoholic) stimuli in peripheral locations for problem drinkers. Appendix B contains further details on the main analyses reported.

\section{Discussion}

The aim of this study was to examine attentional bias in problem and non-problem drinkers using a modified version of a gaze contingency paradigm. This measured eye movements away from a stipulated fixation point towards varying stimuli (termed break frequency). Participants were instructed not to look at the distractor items, be they alcoholic (e.g. wine), non-alcoholic appetitive (e.g., cola) or non-appetitive (e.g., fabric softener). Our findings suggest that when items were presented peripherally to the target (as opposed to central/adjacent), problem drinkers had a higher break frequency towards non-alcoholic stimuli and alcoholic stimuli relative to non-appetitive stimuli. In other words, problem users 
were more likely to break their central focus and produce overt attentional shifts towards both alcoholic and non-alcoholic appetitive stimuli when said stimuli were in the periphery of their vision. Overt attentional shifts were not observed for central stimuli, which may imply that inhibitory control is improved when covert attentional processing is possible. These results are discussed in more detail below.

The difference between problem and non-problem drinkers' performance on this task are in keeping with those of Wilcockson and Pathos (2015), who found that break frequencies to alcohol-related stimuli were higher in heavy (relative to light) drinkers and further supports the notion that there are different attentional biases amongst problem and non-problem drinkers (Sharma, Albery, \& Cook, 2001). However, the current research methods and associated findings are distinct from Wilcockson and Pathos (2015) for two reasons.

First, the current research looked at problem drinkers relative to less problematic drinkers using the AUDIT diagnostic criteria, whereas Wilcockson and Pathos (2015) divided participant into heavy and light drinkers based on self-reported weekly consumption. While there is a degree of overlap in these measures, as heavy drinking is part of the assessment criteria within AUDIT, there is an important distinction in that not all heavy drinkers are problem drinkers. While the results of the current study are therefore broadly equivalent to those of Wilcockson, is it important to highlight the distinction in the populations represented.

Second, Wilcockson and Pothos (2015) utilised neutral, non-appetitive cues and did not measure stimuli location, whereas the current study adopted matched non-alcoholic and nonappetitive cues and compared distraction from central and peripheral cues. Accordingly, we did not find a difference between alcoholic and non-alcoholic items, in terms of break frequency, as was found by Wilcockson and Pathos (2015). As previously argued, this may be the product of the different stimuli used. Indeed, the current findings may suggest that previous work in this area may exaggerate the apparent effect of alcohol-related stimuli by comparing appetitive with non-appetitive cues. Certainly, this assertion appears to be in line with the apparent spill 
over effect found in research using the stop signal task, where decreases in inhibitory control in response to alcohol-related cues appeared to generalise to other appetitive liquids (but not to non-appetitive cues; Monk et al., 2017).

For problem drinkers, peripherally presented cues that were appetitive (alcohol and non alcohol-related cues) resulted in equivalent break frequencies, while both were higher than for non-appetitive cues. This is broadly akin to wider research in this area (see Monk et al., 2017 using a student sample) and may be postulated to be the product of an uncertainty reduction process (Luck, Hillyard, Mouloua, \& Hawkins, 1996; Luck \& Thomas, 1999), whereby attention may operate by reducing uncertainty about which location contains the target. Here, problem drinkers may also be drawn by conditioned associations because alcohol is often served and consumed within soft drinks (i.e., orange juice could contain vodka, for instance), whereas this is clearly not the case for the non-appetitive cues used in this current research (e.g., washing up liquid). Such an assertion appears to be supported by Wiers et al. (2007), who theorise that addictive behaviours, such as alcohol misuse, develop because the appetitive system becomes sensitised with repeated consumption, leading to approach behaviours. Due to the paucity of research in this area, future studies would benefit from assessing whether appetitive conditioning drives attentional bias towards both alcoholic and non-alcoholic appetitive cues. Nevertheless, the current findings may highlight the importance of considering more closely the constitution and presentation location of testing stimuli, alongside the populations of interest.

That the current study found that problem and non-problem drinkers showed no significant differences in eye movements towards centrally located distractors is also potentially interesting from a diagnostic perspective. However, we should note that a lack of eye movement does not necessarily mean a lack of attention (Shepherd, Findlay, \& Hockey, 1986). One interpretation of the lack of eye movements for central stimuli is that these are being processed covertly. Another possibility is that these stimuli may not be processed at all, 
and the current paradigm does not allow us to tease apart this distinction. Nevertheless, as there are eye movements for peripheral stimuli, which indicate a shift of attention, it is probable that the same items presented centrally are also attended to, despite a lack of eye movement. Therefore, covert processing of the central stimuli is more likely (e.g., Riggio \& Kirsner, 1997). We suggest that researchers continue to explore ways that they may further unpick differences in the nature of covert and overt attention for different stimuli types and for problem and non-problem drinkers.

There are limitations to be noted for the current study. First, while the images used were matched as closely as possible on valence and arousal, the valence of non-appetitive stimuli was lower than that of both appetitive stimuli types. Though the arousal scores were relatively similar across stimuli types, the difference in valence may partially explain the differences in break frequencies found between appetitive and non-appetitive stimuli (see Ono \& Taniguchi, 2017). Second, though image sizes were matched, the colours of the images differed. Specifically, some of the non-appetitive stimuli were noticeably more colourful and hence potentially more attractive of gaze (e.g., Frey, Honey, \& König, 2008), though stimuli did not differ on brightness (see Appendix A). Mean break frequencies in general were in fact higher for appetitive stimuli and so the authors were satisfied that colour differences were not the cause of the current study. Nevertheless, it may be beneficial for future research to apply more robust controls over stimuli appearance. Furthermore, it may be fruitful for future explorations to take a measure of baseline thirst, in order to control for the possibility that thirst caused attention towards the appetitive stimuli. Finally, we note that this the current research is based on just one measure of attentional bias and it is unclear whether the same results would be expected to generalize to a more standard measure of attentional bias, such as the Stroop task or the dot probe task. We advocate for further research in this area, expanding the scope of the tasks utilised. 
In sum, the study showed that in contrast with non-problem drinkers, those with problematic alcohol consumption found it more difficult to withhold their attention from appetitive stimuli, particularly when these were in the periphery of their vision. That this occurred for both alcoholic and non-alcoholic stimuli (relative to non-appetitive cues) is interesting and suggests that the appetitive nature of stimuli is a potentially important consideration for research in this area. We tentatively suggest that current results also suggest that attentional biases in problem drinkers may be particularly salient when appetitive stimuli are not directly in their field of view and that an uncertainty reduction processes may explain this. Further research in this area is recommended. 


\section{References}

Adams, S., Ataya, A. F., Attwood, A. S., \& Munafò, M. R. (2013). Effects of alcohol on disinhibition towards alcohol-related cues. Drug and Alcohol Dependence, 127(1), 137142.

Christiansen, P., Cole, J. C. \& Field, M. (2012). Ego depletion increases ad-lib alcohol consumption: investigating cognitive mediators and moderators. Exp Clin Psychopharmacol, 20(2): 118 - 28

Cox, W.M., Yeates, G.N. \& Regan, C.M. (1999). Effects of alcohol cues on cognitive processing in heavy and light drinkers. Drug Alcohol Depend, 55(1-2): 85 - 86

Field, M., Mogg, K., Zetteler, J. \& Bradley, B.P. (2004). Attentional biases for alcohol cues in heavy and light drinkers: the roles of initial orienting and maintained attention. Psychopharmacology, 176(1): 88 - 93

Fleming, K.A. \& Bartholow, B. D. (2014). Alcohol Cues, Approach Bias, and Inhibitory Control: Applying a Dual Process Model of Addiction to Alcohol Sensitivity. Psychol Addict Behav, 28(1): 85 - 96

Frey, H-P., Honey, C. \& König, P. (2008). What's color got to do with it? The influence of color on visual attention in different categories. Journal of Vision, 8(14): 6, 1 - 17

Hogarth L., Dickinson A., Janowski M., Nikitina A., Duka T. (2008). The role of attentional bias in mediating human drug seeking behaviour. Psychopharmacology, 201:29-41.

Inzlicht, M., \& Kang, S. K. (2010). Stereotype threat spillover: how coping with threats to social identity affects aggression, eating, decision making, and attention. Journal of personality and social psychology, 99(3), 467-481.

Jones, A., \& Field, M. (2015). Alcohol-related and negatively valenced cues increase motor and oculomotor disinhibition in social drinkers. Experimental and Clinical Psychopharmacology, 23(2), 122-129. 
Jones, A., Di Lemma, L. C., Robinson, E., Christiansen, P., Nolan, S., Tudur-Smith, C., \& Field, M. (2016). Inhibitory control training for appetitive behaviour change: A metaanalytic investigation of mechanisms of action and moderators of effectiveness. Appetite, 97, 16-28.

Jones, A., Rose, A. Cole, J. \& Field, M. (2013). Effects of Alcohol Cues on Craving and Ad Libitum Consumption in Social Drinkers: The Role of Disinhibition. Journal of Experimental Psychopathology, 4(3): 239 - 249

Kreusch, F., Vilenne, A., \& Quertemont, E. (2013). Response inhibition toward alcohol related cues using an alcohol go/no-go task in problem and non-problem drinkers. Addictive Behaviors, 38(10), 2520-2528.

Lang, P. J. (1980). Behavioral treatment and bio-behavioral assessment: Computer applications. In J. B. Sidowski, J. H. Johnson, \& T. A. Williams (Eds.), Technology in mental health care delivery systems (pp. 119-137). Norwood, NJ: Ablex.

Li, C. S. R., Luo, X., Yan, P., Bergquist, K., \& Sinha, R. (2009). Altered impulse control in alcohol dependence: neural measures of stop signal performance. Alcoholism: Clinical and Experimental Research, 33(4), 740-750.

Leotti, L. A., \& Wager, T. D. (2009). Motivational influences on response inhibition. Journal of Experimental Psychology: Human Perception \& Performance, 36, 430-447.

Luck, S. J., Hillyard, S. A., Mouloua, M. \& Hawkins, H. L. (1996). Mechanisms of VisualSpatial Attention: Resource Allocation or Uncertainty Reduction? Journal of Experimental Psychology: Human Perception and Performance. 22(3): 725 - 737

Monk, R.L., Sunley, J., Qureshi, A.W., \& Heim, D. (2016). Smells like inhibition: The effects of olfactory and visual alcohol cues on inhibitory control. Psychopharmacology, 233, 1331-1337.

Monk, R.L., Qureshi, A., Pennington, C.R., \& Hamlin, I. (2017). Generalised inhibitory impairment to appetitive cues: From alcoholic to non-alcoholic visual stimuli. Drug and Alcohol Dependence. 
Muraven, M., Baumeister, R. F. (2000). Self-regulation and depletion of limited resources: Does self-control resemble a muscle? Psychological Bulletin, 126(2), 247-259.

Nederkoorn, C., Baltus, M., Guerrieri, R., \& Wiers, R. W. (2009). Heavy drinking is associated with deficient response inhibition in women but not in men. Pharmacology Biochemistry \& Behavior, 93(3), 331-336.

Ono, Y. \& Taniguchi, Y. (2017). Attentional Capture by Emotional Stimuli: Manipulation of Emotional Valence by the Sample Pre-rating Method. Japanese Psychological Research, 59(1): $26-34$

Pronk, T., van Deursen, D. S., Beraha, E. M., Larsen, H. \& Wiers, R. W. (2015). Validation of the Amsterdam Beverage Picture Set: A Controlled Picture Set for Cognitive Bias Measurement and Modification Paradigms. Alcoholism: Clinical and Experimental Research, 19(10): 2047 - 2055

Riggio, L. \& Kirsner, K. (1997). The relationship between central cues and peripheral cues in covert visual orientation. Perception \& Psychophysics, 59(6): 885 - 899

Rose, A.K. \& Duka, T. (2008). Effects of alcohol on inhibitory processes. Behav Pharmacol, 19(4): 284 - 91

Qureshi. A.W., Monk, R.L., Pennington, C.R., Li, X., \& Leatherbarrow, T. (2017). Context and alcohol consumption behaviours affect inhibitory control. Journal of Applied Social Psychology, 47 (11): 625-633

Saunders, J. B., Aasland, O. G., Babor, T. F., De la Fuente, J. R., \& Grant, M. (1993). Development of the alcohol use disorders identification test (AUDIT). WHO collaborative project on early detection of persons with harmful alcohol consumptionII. Addiction, 88, 791-791.

Sharma, D., Albery, I.P. \& Cook, C. (2001). Selective attentional bias to alcohol related stimuli in problem drinkers and non-problem drinkers. Addiction, 96(2): 285 - 95 
Shepherd, M., Findlay, J.M. \& Hockey, R.J. (1986). The relationship between eyemovements and spatial attention. Quarterly Journal of Experimental Psychology Section A - Human Experimental Psychology, 38 (3): 475-491

Simpson, A., \& Riggs, K. J. (2006). Conditions under which children experience inhibitory difficulty with a "button-press" go/no-go task. Journal of Experimental Child Psychology, 94(1), 18-26.

Weafer, J., \& Fillmore, M. T. (2015). Alcohol-related cues potentiate alcohol impairment of behavioral control in drinkers. Psychology of Addictive Behaviors, 29(2), 290-299.

Wilcockson, T. D. W., \& Pothos, E. M. (2015). Measuring inhibitory processes for alcoholrelated attentional biases: Introducing a novel attentional bias measure. Addictive behaviors, 44, 88-93. 